\title{
Second Generation Needling Techniques for the Treatment of Chondral Defects in Animal Model
}

\author{
Pietro Zedde ${ }^{1}$ Sebastiano Cudoni ${ }^{1}$ Lucia Manunta ${ }^{2}$ Eraldo Sanna Passino ${ }^{2}$ Gerolamo Masala ${ }^{2}$ \\ Antonio Brunetti ${ }^{3}$ Francesco Mattia Uboldi ${ }^{4}$ Andrea Fabio Manunta ${ }^{4}$
}

1 Orthopedic and Traumatology Unit, Nuoro Hospital, Nuoro, Italy
2 Department of Veterinary Surgery, University of Sassari,
Sassari, Italy
3 Polcoming Department, University of Sassari, Sassari, Italy
${ }^{4}$ Orthopedic Traumatology Unit, Department of Biomedical Science,
Sassari University Hospital, Sassari, Italy

Joints 2017;5:27-33.

\author{
Address for correspondence Andrea Fabio Manunta, MD, Orthopedic \\ Traumatology Unit, Department of Biomedical Science, Sassari \\ University Hospital, Viale San Pietro 43, 07100 Sassari, Italy \\ (e-mail: pzedde53@gmail.com).
}

\begin{abstract}
Keywords

- microfracture

- nanofracture

- articular cartilage repair

- mesenchymal stem cells

- sheep

Purpose To compare the macroscopic, histological, and immunohistochemical characteristics of the repair tissue of chondral defects treated with microfracture and nanofracture in an ovine model.

Methods Full-thickness chondral lesions were created in the medial femoral condyle of both knees in four adult sheep and were treated with microfracture on one side and with nanofracture on the contralateral side. Chondral repair was assessed after 12 months by macroscopic, histological, and immunohistochemical analyses.

Results Histological cartilage repair significantly improved in the samples treated with nanofracture for cellular morphological characteristics and cartilage architecture. The immunohistochemical analysis showed a significantly higher immunoreactivity to type II collagen in the defects treated with nanofracture.

Conclusion Nanofracture provided better repair tissue than microfracture, with a more satisfactory cartilage architecture renovation and tissue having greater type II collagen content.

Clinical Relevance Mesenchymal stem cell stimulation is the most frequently used primary cartilage repair procedure. Nanofracture represents a novel technique to stimulate bone marrow that results into a successful repair of chondral defects.
\end{abstract}

\section{Introduction}

Although almost 60 years have passed since the description of the first bone marrow stimulation technique by Smillie, ${ }^{1}$ the treatment of chondral lesions so far represents a relevant and challenging issue. Despite the fact that along the years and especially in the recent past several treatments and novel biomaterials have been proposed, none of the different methods available has been able to renovate the joint surface from both the biological and biomechanical standpoint. ${ }^{2}$ The microfracture technique developed by Steadman still represents the most frequently used procedure. ${ }^{3}$ Approximately 80,000 mi- crofracture procedures are performed every year in the United States. ${ }^{4,5}$ The biological rationale of bone marrow stimulation is attributed to the role of mesenchymal stem cells (MSCs) recruited from the bone marrow. ${ }^{6-11}$ The advantages of this technique include its minimal invasiveness, favorable costeffectiveness ratio, and low technical demands. Satisfactory results are being achieved especially in young patients with defects of limited size., ${ }^{8,12}, 13$ However, the quality and characteristics of the newly formed fibrocartilaginous tissue have been widely discussed in the literature ${ }^{14,15}$ due to the limited biomechanical properties of the tissue rich in type I collagen and due to its tendency to degenerate. 
In recent years, improved knowledge and understanding of the structural and functional architecture of subchondral bone has led to an increased focus on the need to preserve its integrity during chondral defect treatment.

The limited penetration and the broad diameter of the $\mathrm{awl}^{16}$ are currently thought to play a key role in repairing fibrocartilaginous tissue, as well as in subchondral bone compaction around the perforation ${ }^{9,16,17}$ and in intralesional osteophytes formation. ${ }^{9,18}$ To overcome the mechanical limitations of microfracture, the nanofracture technique was recently introduced using smaller diameter and deeper subchondral bone needle perforations (Arthrosurface Inc., Franklin, Massachusetts, United States). ${ }^{9}$

The aim of this study was to assess macroscopic, histological, and immunohistochemical characteristics of repair tissue of chondral defects treated with microfractures or nanofractures in an ovine model. The hypothesis of the study was that nanofracture provides better repair tissue than microfracture.

\section{Methods}

The local ethic committee approved this study, and all procedures were conducted according to the Institutional Animal Care regulations, which conformed to the National Institutes of Health Guidelines on the Care of Laboratory Animals.

Four adult Sardinian ewes, aged 5.5 years, weighing approximately $45 \mathrm{~kg}$, were used in the study. All animals were examined and found to be in good health.

Surgery was performed under sterile conditions and with sheep under general anesthesia. All sheep were intubated after the administration of thiopentone $(25 \mathrm{mg} / \mathrm{kg})$ and ventilated with $\mathrm{O}_{2}$ in $\mathrm{N}_{2} \mathrm{O}$ by volume control. Anesthesia was maintained with 1.5 to $2 \%$ of isoflurane; a bolus dose of $0.1 \mathrm{mg}$ of fentanyl was given before surgery.

A medial parapatellar arthrotomy was performed on the right and left stifle of all animals. Incision was performed to expose the medial femoral condyle in both hind legs. An 8mm-diameter (area: $50.3 \mathrm{~mm}^{2}$ ), full-thickness chondral lesion in the load-bearing area of each medial femoral condyle was created using an arthroscopic burr. The calcified cartilage layer was removed; vertical walls were created at the periphery of the cartilage lesion. The defects were then treated with microfracture on one side and nanofracture on the contralateral side. Each cartilage lesion was treated with five bone channels. The distance between each channel was $3 \mathrm{~mm}$ according to previous recommendations. ${ }^{7}$ Microfracture sites were treated using an awl manufactured by a curved Steadman awl. The perforation depth was user-controlled with visual feedback from the awl tip. Nanofracture sites were treated using a cannulated awl and a 1-mm-thick Nitinol needle (NanoFx, Arthrosurface Inc). The 9-mm perforation depth of the needle was controlled by the awl (-Fig. 1). The surgical technique was previously described. ${ }^{9}$ Upon completion of the cartilage repair procedures, all incisions were closed in layers according to standard surgical practice. Postoperatively, the animals were kept in stalls with limited movement and weight-bearing. Animals were then left free to roam in their fencings without any immobilization of the operated limb. Full weight-bearing was allowed as tolerated, and no specific exercise regimen was adopted. General health and weight-bearing status were monitored by a veterinary during recovery.

Immediately after euthanasia, the defects were photographed to allow assessment by two blinded observers. Macroscopic evaluation was assessed based on the International Cartilage Repair Society (ICRS) evaluation score (-Table 1).

Condylar articular defects containing regenerated tissue, adjacent host cartilage, and subchondral bone were harvested using a water-cooled circular saw. The tissue blocks were fixed in $10 \%$ neutral-buffered formalin for 4 days and then placed in a decalcification solution for 4 to 10 days. After washing in running tap water for 4 to 8 hours to remove all traces of decalcification solution, the osteochondral specimens were paraffin-embedded and $4-\mu$ sections were stained with safranin orange/fast green (Safranin 0) and hematoxylin and eosin as previously described. ${ }^{19}$

For the immunohistochemical analysis, we used the avidin-biotin complex and peroxidase. The immunohistochemical determination of type I and type II collagen contents was conducted on paraffin-embedded sections using an automatic immunostainer (Ventana BenchMark ULTRA, Roche

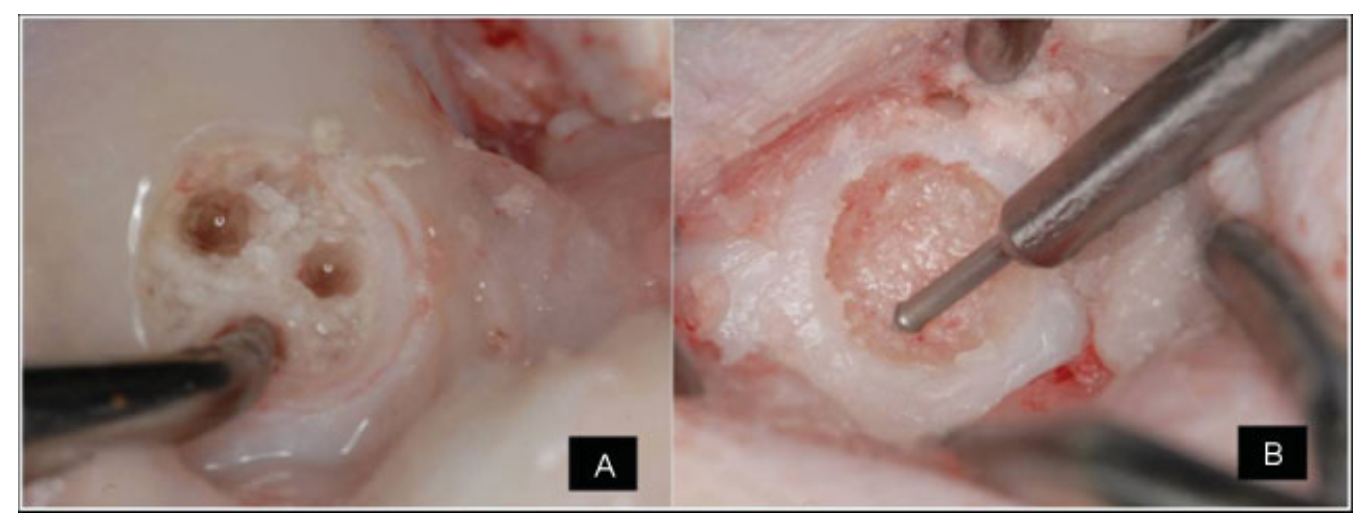

Fig. 1 (A) Treatment of the defect with microfracture. (B) Treatment of the defect with nanofracture. 
Table 1 International Cartilage Repair Society macroscopic evaluation of cartilage repair

\begin{tabular}{|c|c|}
\hline Categories & Score \\
\hline \multicolumn{2}{|l|}{ Degree of defect repair } \\
\hline In level with surrounding cartilage & 4 \\
\hline $75 \%$ repair of defect depth & 3 \\
\hline $50 \%$ repair of defect depth & 2 \\
\hline $25 \%$ repair of defect depth & 1 \\
\hline $0 \%$ repair of defect depth & 0 \\
\hline \multicolumn{2}{|l|}{ Integration to border zone } \\
\hline Complete integration with surrounding cartilage & 4 \\
\hline Demarcating border $<1 \mathrm{~mm}$ & 3 \\
\hline $3 / 4$ of graft integrated, $1 / 4$ with a notable border $>1 \mathrm{~mm}$ width & 2 \\
\hline $1 / 2$ of graft integrated with surrounding cartilage, $1 / 2$ with a notable border $>1 \mathrm{~mm}$ & 1 \\
\hline From no contact to $1 / 4$ of graft integrated with surrounding cartilage & 0 \\
\hline \multicolumn{2}{|l|}{ Macroscopic appearance } \\
\hline Intact smooth surface & 4 \\
\hline Fibrillated surface & 3 \\
\hline Small, scattered fissures or cracs & 2 \\
\hline Several, small or few but large fissures & 1 \\
\hline Total degeneration of grafted area & 0 \\
\hline \multicolumn{2}{|l|}{ Overall repair assessment } \\
\hline Grade I: normal & 12 \\
\hline Grade II: nearly normal & $11-8$ \\
\hline Grade III: abnormal & $7-4$ \\
\hline Grade IV: severely abnormal & $3-1$ \\
\hline
\end{tabular}

Diagnostics, Basel, Switzerland), with a ready-to-use dilution of a monoclonal mouse antitype I or antitype II collagen immunoglobulin (ThermoFisher Scientific, Waltham, Massachusetts, United States). The reaction was displayed by the Ultraview DAB kit (Ventana Medical System, Roche Diagnostics), a revelation system kit including the secondary antimouse antibody biotinylated, the enzymatic substrate, and the chromogen DAB.

Immunoreactivity to type I collagen in the repair tissue was compared with that of the adjacent subchondral bone, serving as positive internal control $(0=$ no immunoreactivity; 1 = significantly weaker; $2=$ moderately weaker; $3=$ similar; $4=$ stronger immunoreactivity).

\section{Results}

\section{Macroscopic Evaluation}

Macroscopic evaluations conducted according to the ICRS evaluation score on chondral defects treated with microfracture technique showed a partial filling of the defect by a thin healing tissue; the latter showed characteristics similar to the healthy cartilage almost exclusively on the peripheral areas of the lesion (-Fig. 2). Conversely, macroscopic evaluations performed on condyles treated by using the

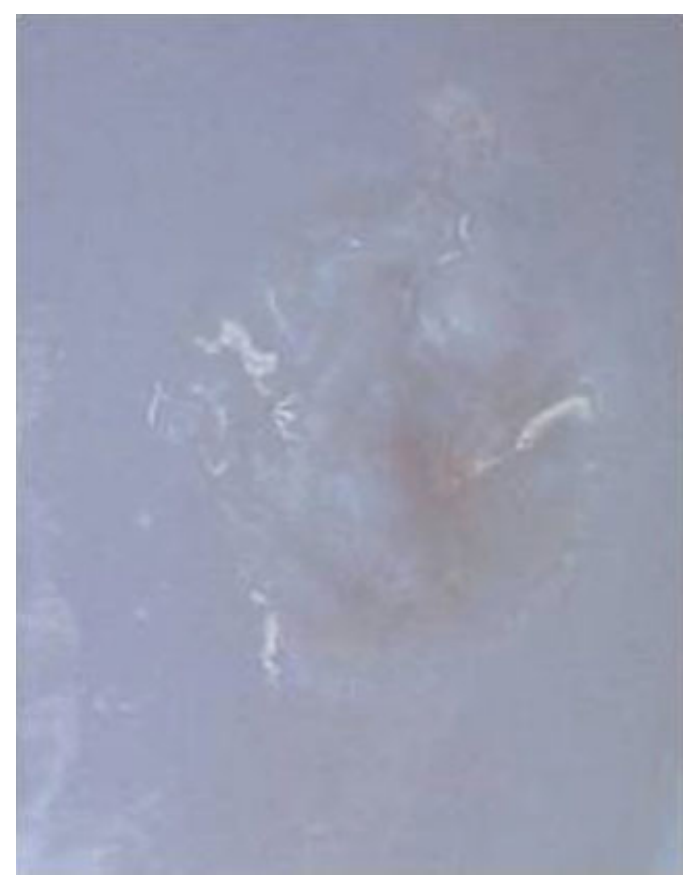

Fig. 2 Macroscopic appearance of a defect treated with microfracture technique 12 months after surgery. Partial filling of the defect by a thin healing tissue can be observed. 


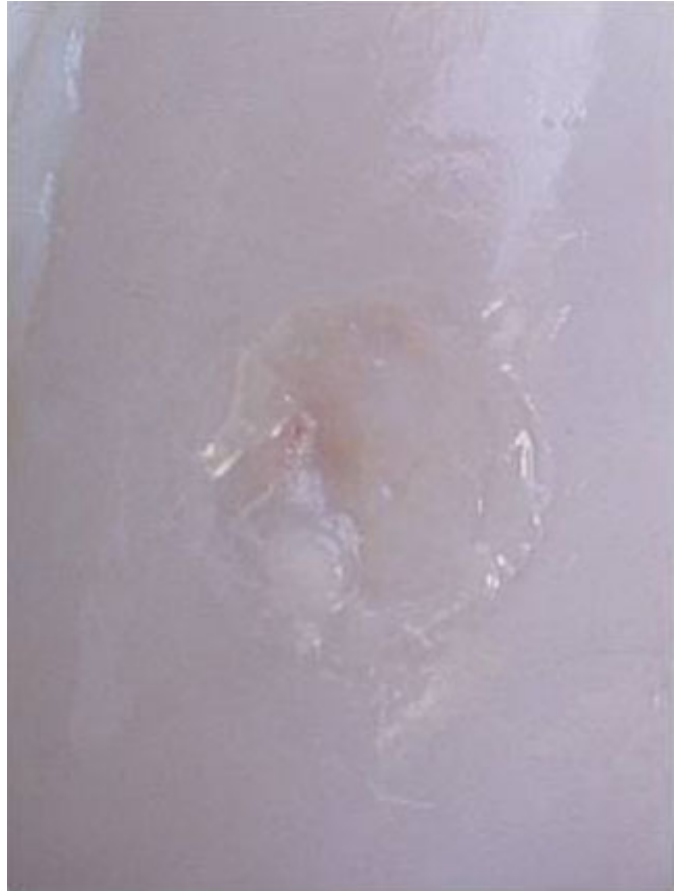

Fig. 3 Macroscopic appearance of a defect treated with nanofracture technique 12 months after surgery. The defect is almost completely covered by a newly formed tissue that is similar to the native cartilage. Furthermore, the repair tissue shows a good integration with the surrounding cartilage and lack of fibrillation and fissures on the majority of the surface.

nanofracture technique showed an almost complete covering of the defect by a newly formed tissue, resulting more similar to the native cartilage in the whole chondral defect. Furthermore, the repair tissue showed a higher integration with the surrounding cartilage and lack of fibrillation and fissures on the majority of the surface ( - Fig. 3 ).

\section{Histological and Immunohistochemical Evaluation}

Histological evaluation on samples treated using the microfracture technique showed a partial filling of the defect by fibrocartilaginous tissue formed by rounded cells similar to chondrocytes submerged into a fibrous extracellular matrix. The repair tissue did not show the normal structure consisting of cartilage layers and was characterized by an almost complete lack of tidemark and a severe alteration of the subchondral bone architecture (-Fig. 4).

On the other hand, the histological evaluation of samples treated with the nanofracture technique highlighted a more satisfactory defect filling and a better structural cartilage architecture into the lesion. The newly formed tissue showed the same features as a fibrohyaline repair tissue in which small groups of rounded cellular elements clustered and diffusely distributed could be observed (

Immunofluorescence showed a scarce positivity for type II collagen in defects treated with microfractures (-Fig. 6) compared with defects treated by with nanofractures. In the latter group, the repair tissue presented a strong positivity for type II collagen, visible especially around the perforations (-Fig. 7).

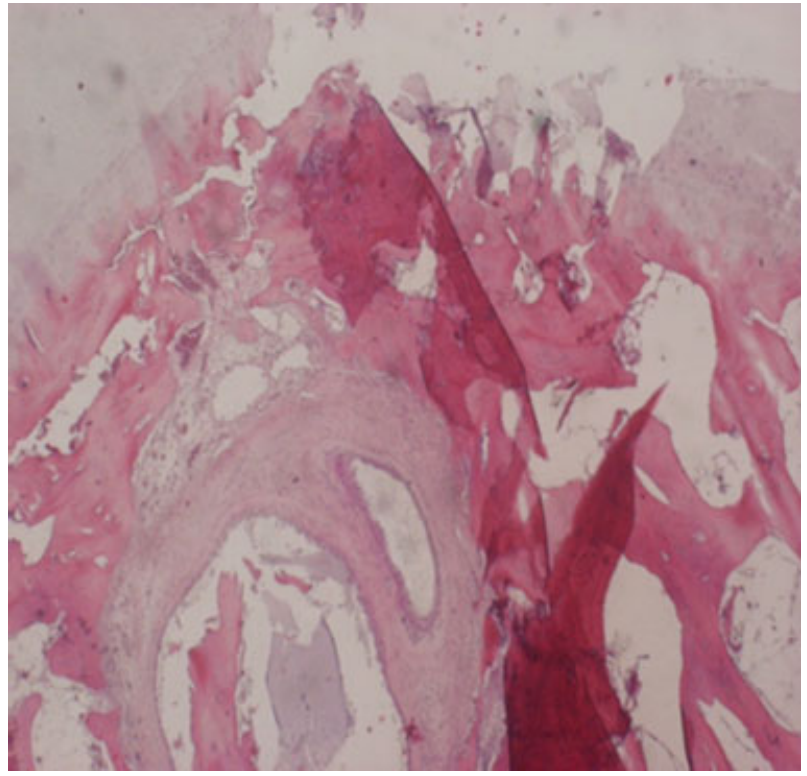

Fig. 4 Histological evaluation of a defect treated with microfracture technique 12 months after surgery. Repair tissue does not show the normal structure consisting of cartilage layers and is characterized by an almost complete lack of tidemark and a severe alteration of the subchondral bone architecture.

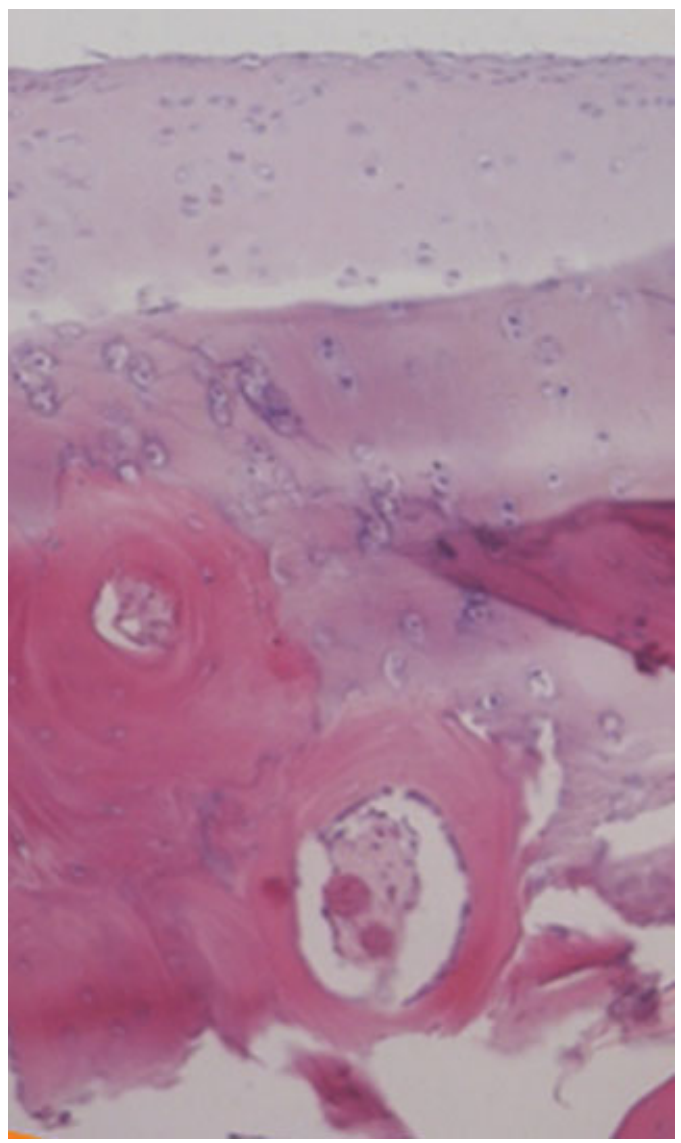

Fig. 5 Histological evaluation of a defect treated with nanofracture technique 12 months after surgery. Satisfactory defect filling and restoration of the structural cartilage architecture can be observed. 


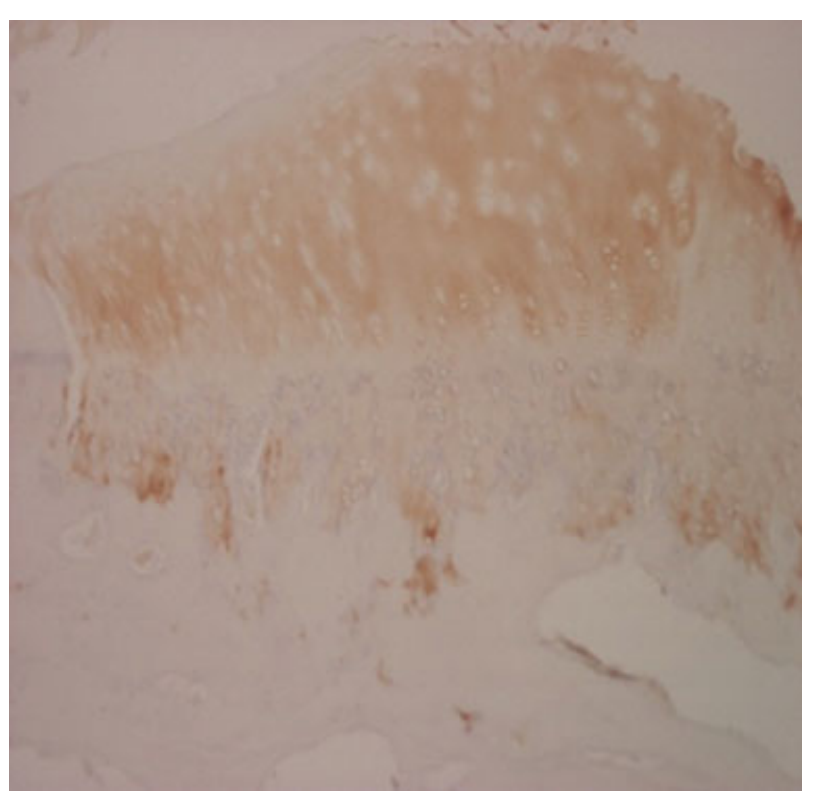

Fig. 6 Immunohistochemical evaluation of a defect treated with microfracture technique 12 months after surgery. The defect is filled with fibrocartilage tissue, with poor immunofluorescence positivity for type II collagen.

\section{Discussion}

The main finding of this study is that small-diameter needle perforation into full-thickness chondral defects significantly improves repair in a preclinical animal model at 12 months postoperatively compared with large drill holes.

Since the first use proposed by Steadman in the 1980s, microfracture has become the treatment of choice for the management of chondral lesions. ${ }^{3}$ The technique is based on direct stimulation of MSCs of the subchondral bone, which have a high potential for differentiation into various connective tissues, including cartilage, bone, tendon, and ligaments. ${ }^{20,21}$ The regenerative effects of MSCs achieve their objective in promoting tissue repair and the resolution of inflammation through direct cell-to-cell interaction or by secretion of bioactive components. ${ }^{22,23}$ Several studies confirmed the efficacy of the microfracture technique, with improved clinical outcomes in 70 to $90 \%$ of patients. ${ }^{7,8,13,24-26}$ However, the formation of fibrocartilage tissue and the deterioration of clinical results over time represent important limitations, ${ }^{27-30}$ and alternative procedures for the management of chondral lesions have been advocated. ${ }^{14,15}$

The use of standard microfracture awls is no longer supported by recent basic science evidence. ${ }^{17,31,32}$

Eldracher et al conducted a study on an animal model aiming at evaluating through macroscopic, histological, immunohistochemical, and microcomputed tomography analysis the repair of chondral defects treated by a perforation diameter of either 1 or $1.8 \mathrm{~m}$. Results were definitely better in defects treated with smaller diameter holes, repair tissue having greater content in type II collagen and showing an architecture more similar to normal. ${ }^{33}$

Orth et al recently published their comparative results treating $8 \times 4$-mm full-thickness defects in the weightbearing area of ovine knees. The study used two custommade awls with a diameter of either 1 or $1.2 \mathrm{~mm}$. The perforation depth was stop controlled at $5 \mathrm{~mm}$; the distal tips were trihedral. The authors reported a significant improvement in the overall histological score using small diameter awls. In addition, the histological surface regularity was significantly improved by smaller instruments. ${ }^{32}$

Chen et al published several studies evaluating the histological characteristics of the newly formed tissue after treatment of chondral defects with different reparative techniques and various degrees of penetration into the subchondral bone. ${ }^{17,31,34}$ Histological and histomorphometric evaluations showed that the best access to bone marrow was observed when drilling to greater depths, thereby improving defect fill and production of cartilage with a higher hyaline content. ${ }^{25} \mathrm{~A}$ further study by the same group showed that treatment with microfracture is associated with fragmentation and compaction around the trabecular canals. ${ }^{17}$

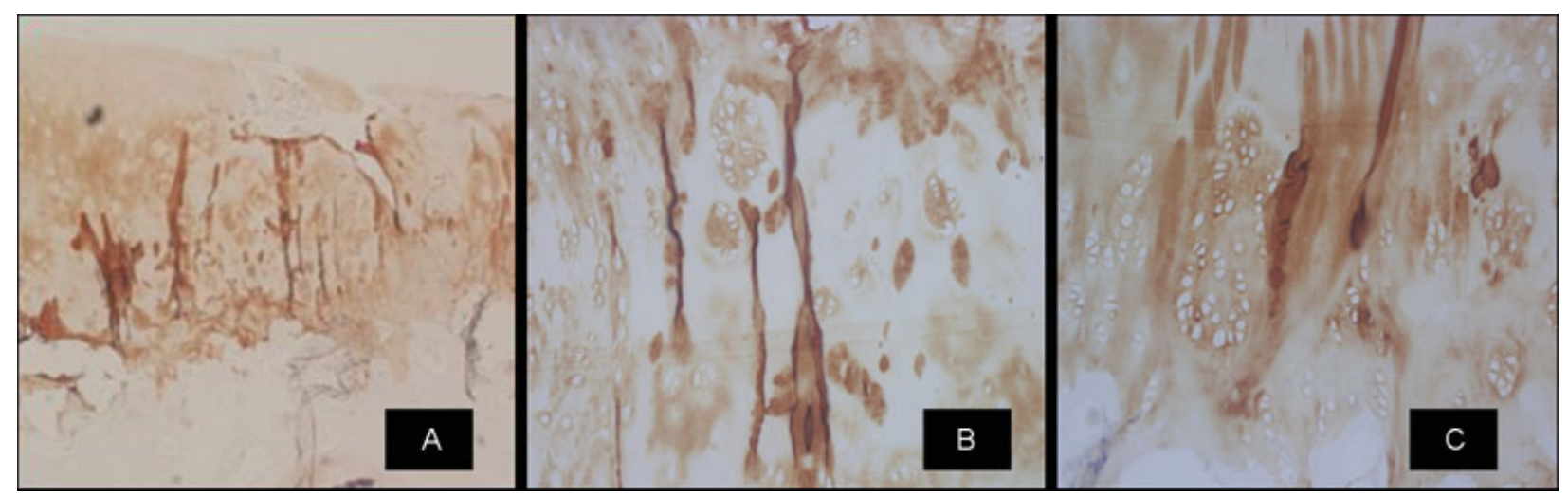

Fig. 7 Immunohistochemical evaluation of a defect treated with nanofracture technique 12 months after surgery. (A) The defect is completely filled by fibrohyaline repair tissue strongly positive to immunohistochemistry for type II collagen. (B) Strong positivity in correspondence of the channels can be observed. (C) Clones of regenerating chondrocytes are present around the area that correspond to increased collagen type II synthesis. 
Our study confirmed previous results, showing beneficial subchondral effects using small diameter needle perforation. While Orth et $\mathrm{al}^{32}$ chose a 5 -mm perforation depth using custom instruments, we used clinically available instrumentation for nanofracture involving the use of a 1-mm-thick needle inserted into a cannulated awl that creates stopcontrolled 9-mm-deep perforations. This technique was compared with microfracture using the standard conically shaped tip.

In accordance with different studies published in the literature, our work highlights how chondral defects repair obtained with reduced diameter perforations and with a higher penetration into the subchondral bone is associated with the formation of tissue having histological and immunohistological characteristics more similar to the native cartilage compared with standard microfracture. Better results obtained with nanofracture might be explained by a lower fragmentation and trabecular compaction around the perforations, leading to a better communication between those and the surrounding trabecular channels. These canals allow for the immediate on-site formation of a superclot, resulting in improved recruitment of multipotent MSCs.

Overall, inference of an animal study is limited because functional status cannot be assessed at baseline and followup evaluations. However, the animal model used in this study is considered suitable for cartilage defect testing. Limitations due to small cohort size should also be considered.

Finally, no biomechanical analysis of the newly formed tissue was performed.

In conclusion, nanofracture represents a novel technique for bone marrow stimulation based on smaller diameter and deeper perforations, resulting into a more satisfactory chondral repair compared with methods based on perforation of larger diameter and lower depth.

\section{References}

1 Smillie IS. Treatment of osteochondritis dissecans. J Bone Joint Surg Br 1957;39-B(02):248-260

2 Mazor M, Lespessailles E, Coursier R, Daniellou R, Best TM, Toumi $\mathrm{H}$. Mesenchymal stem-cell potential in cartilage repair: an update. J Cell Mol Med 2014;18(12):2340-2350

3 Steadman JR, Rodkey WG, Rodrigo JJ. Microfracture: surgical technique and rehabilitation to treat chondral defects. Clin Orthop Relat Res 2001;(391 Suppl):S362-S369

4 McCormick F, Harris JD, Abrams GD, et al. Trends in the surgical treatment of articular cartilage lesions in the United States: an analysis of a large private-payer database over a period of 8 years. Arthroscopy 2014;30(02):222-226

5 Montgomery SR, Foster BD, Ngo SS, et al. Trends in the surgical treatment of articular cartilage defects of the knee in the United States. Knee Surg Sports Traumatol Arthrosc 2014;22(09): 2070-2075

6 Khan WS, Johnson DS, Hardingham TE. The potential of stem cells in the treatment of knee cartilage defects. Knee 2010;17(06): 369-374

7 Mithoefer K, Williams RJ III, Warren RF, et al. The microfracture technique for the treatment of articular cartilage lesions in the knee. A prospective cohort study. J Bone Joint Surg Am 2005; 87(09):1911-1920
8 Mithoefer K, Williams RJ III, Warren RF, et al. Chondral resurfacing of articular cartilage defects in the knee with the microfracture technique. Surgical technique. J Bone Joint Surg Am 2006;88 (Suppl 1 Pt 2):294-304

9 Benthien JP, Behrens P. Reviewing subchondral cartilage surgery: considerations for standardised and outcome predictable cartilage remodelling: a technical note. Int Orthop 2013;37(11): 2139-2145

10 Min BH, Choi WH, Lee YS, et al. Effect of different bone marrow stimulation techniques (BSTs) on MSCs mobilization. J Orthop Res 2013;31(11):1814-1819

11 Shapiro F, Koide S, Glimcher MJ. Cell origin and differentiation in the repair of full-thickness defects of articular cartilage. J Bone Joint Surg Am 1993;75(04):532-553

12 Kreuz PC, Steinwachs MR, Erggelet C, et al. Results after microfracture of full-thickness chondral defects in different compartments in the knee. Osteoarthritis Cartilage 2006;14(11): 1119-1125

13 Mithoefer K, Williams RJ III, Warren RF, Wickiewicz TL, Marx RG. High-impact athletics after knee articular cartilage repair: a prospective evaluation of the microfracture technique. Am J Sports Med 2006;34(09):1413-1418

14 Saris DB, Vanlauwe J, Victor J, et al. Characterized chondrocyte implantation results in better structural repair when treating symptomatic cartilage defects of the knee in a randomized controlled trial versus microfracture. Am J Sports Med 2008; 36(02):235-246

15 Saris DB, Vanlauwe J, Victor J, et al; TIG/ACT/01/2000\&EXT Study Group. Treatment of symptomatic cartilage defects of the knee: characterized chondrocyte implantation results in better clinical outcome at 36 months in a randomized trial compared to microfracture. Am J Sports Med 2009;37(Suppl 1):10S-19S

16 Hoemann CD, Gosselin Y, Chen H, et al. Characterization of initial microfracture defects in human condyles. J Knee Surg 2013;26(05):347-355

17 Chen H, Sun J, Hoemann CD, et al. Drilling and microfracture lead to different bone structure and necrosis during bone-marrow stimulation for cartilage repair. J Orthop Res 2009;27(11): 1432-1438

18 Orth P, Goebel L, Wolfram U, et al. Effect of subchondral drilling on the microarchitecture of subchondral bone: analysis in a large animal model at 6 months. Am J Sports Med 2012;40(04): 828-836

19 Orth P, Cucchiarini M, Kaul G, et al. Temporal and spatial migration pattern of the subchondral bone plate in a rabbit osteochondral defect model. Osteoarthritis Cartilage 2012;20(10): 1161-1169

20 Caplan AI, Dennis JE. Mesenchymal stem cells as trophic mediators. J Cell Biochem 2006;98(05):1076-1084

21 Lodi D, Iannitti T, Palmieri B. Stem cells in clinical practice: applications and warnings. J Exp Clin Cancer Res 2011;30:9. Doi: 10.1186/1756-9966-30-9

22 Caplan AI. Review: mesenchymal stem cells: cell-based reconstructive therapy in orthopedics. Tissue Eng 2005;11(7-8): 1198-1211

23 Chen FH, Tuan RS. Mesenchymal stem cells in arthritic diseases. Arthritis Res Ther 2008;10(05):223. Doi: 10.1186/ar2514

24 Steadman JR, Rodkey WG, Singleton SB, et al. Microfracture technique for full-thickness chondral defects. Technique and clinical results. Oper Tech Orthop 1997;7:300-304

25 Steadman JR, Miller BS, Karas SG, Schlegel TF, Briggs KK, Hawkins RJ. The microfracture technique in the treatment of full-thickness chondral lesions of the knee in National Football League players. J Knee Surg 2003;16(02):83-86

26 Steadman JR, Briggs KK, Rodrigo JJ, Kocher MS, Gill TJ, Rodkey WG. Outcomes of microfracture for traumatic chondral defects of the knee: average 11-year follow-up. Arthroscopy 2003;19(05): 477-484 
27 Kon E, Gobbi A, Filardo G, Delcogliano M, Zaffagnini S, Marcacci M. Arthroscopic second-generation autologous chondrocyte implantation compared with microfracture for chondral lesions of the knee: prospective nonrandomized study at 5 years. Am J Sports Med 2009;37(01):33-41

28 Kon E, Filardo G, Berruto M, et al. Articular cartilage treatment in high-level male soccer players: a prospective comparative study of arthroscopic second-generation autologous chondrocyte implantation versus microfracture. Am J Sports Med 2011;39(12): 2549-2557

29 Mithoefer K, McAdams T, Williams RJ, Kreuz PC, Mandelbaum BR. Clinical efficacy of the microfracture technique for articular cartilage repair in the knee: an evidence-based systematic analysis. Am J Sports Med 2009;37(10):2053-2063

30 Namdari S, Baldwin K, Anakwenze O, Park MJ, Huffman GR, Sennett BJ. Results and performance after microfracture in Na- tional Basketball Association athletes. Am J Sports Med 2009; 37(05):943-948

31 Chen H, Chevrier A, Hoemann CD, Sun J, Ouyang W, Buschmann MD. Characterization of subchondral bone repair for marrowstimulated chondral defects and its relationship to articular cartilage resurfacing. Am J Sports Med 2011;39(08):1731-1740

32 Orth P, Duffner J, Zurakowski D, Cucchiarini M, Madry H. Smalldiameter awls improve articular cartilage repair after Microfracture treatment in a translation animal model. Am J Sports Med 2016;44(01):209-219

33 Eldracher M, Orth P, Cucchiarini M, Pape D, Madry H. Small subchondral drill holes improve marrow stimulation of articular cartilage defects. Am J Sports Med 2014;42(11):2741-2750

34 Chen H, Hoemann CD, Sun J, et al. Depth of subchondral perforation influences the outcome of bone marrow stimulation cartilage repair. J Orthop Res 2011;29(08):1178-1184 